\title{
High Positioning Repeatability of Miniature Actuator
}

\author{
Chien-Sheng Liu* and Psang-Dain Lin \\ Department of Mechanical Engineering, National Cheng Kung University, Tainan 70101, Taiwan
}

(Received July 17, 2008; accepted October 1, 2008)

Key words: actuator, voice coil motor, Hall element, camera module

Autofocusing actuators in camera phones should have a reduced profile and an improved performance in accordance with the requirements of consumer electronic products. In this study, we first discuss the detailed design of the miniature actuator presented in our previous work ${ }^{(10)}$ and then describe an experiment to test its positioning repeatability. As compared with conventional voice coil motor (VCM) actuators positioned by open-loop control, the proposed miniature VCM actuator is small, has a high positioning repeatability and can improve the positioning repeatability by $87.5 \%$ of the typical value.

\section{Introduction}

Recently, the mobile camera phone market has increased markedly, particularly with regard to the integration of high-end camera functions.(1,2) Many studies have contributed to the development of camera modules, for example, voice coil motors (VCMs), piezoelectric motors, and tunable microlenses, for cell phone applications. ${ }^{(3-5)}$ Among these modules, the VCMs are the most promising modules owing to their low cost, low power consumption, fast response, high repeatability, and small size. ${ }^{(6)}$ Furthermore, it has been noted that these features are intrinsically important for future commercial applications. To the best of our knowledge, the studies reported in the literature pertaining VCM actuators focused only on their fast dynamic response and low power consumption. ${ }^{(7-9)}$ Moreover, to satisfy the requirements of an autofocusing camera module, a VCM actuator should have a high positioning repeatability.

To satisfy the requirements of the autofocusing camera module, a miniature VCM actuator (size: $6.5 \times 6.5 \times 4 \mathrm{~mm}^{3}$ ) was developed by our group. ${ }^{(10)}$ In this study, we first discuss the mechanical structure of the VCM actuator and subsequently provide detailed descriptions of its electromagnetic and electronic circuit designs. An experiment will be conducted to investigate the positioning repeatability of the VCM actuator. It is shown that as compared with the existing VCM actuators, the proposed VCM actuator is small $\left(6.5 \times 6.5 \times 4 \mathrm{~mm}^{3}\right)$ and has a high positioning repeatability (less than $\left.5 \mu \mathrm{m}\right)$.

*Corresponding author: e-mail: chienshengliu@itri.org.tw 


\section{Conventional VCM Actuator}

A typical commercial VCM actuator (size: $10 \times 10 \times 4.6 \mathrm{~mm}^{3}$ ) with an open-loop control in the autofocusing camera module for cell phones has two major components, the fixed and moving parts. ${ }^{(3,10)}$ Two spring plates are required to connect these two parts at both sides to provide restoring resilience to balance the Lorentz force $F_{\mathrm{VCM}}$ of the VCM actuator. Figure 1 shows simplified force models of the conventional VCM for the upright, horizontal, and upside-down positions. Furthermore, the restoring force $F_{\mathrm{s}}=\mathrm{k} \delta$ of the spring plates, the Lorentz force $F_{\mathrm{VCM}}$, and the weight force $F_{\mathrm{W}}$ of the moving part are also shown in Fig. 1. Here, $\mathrm{k}$ and $\delta$ are the spring constant of the spring plates and the displacement of the moving part, respectively. $F_{\mathrm{VCM}}$ is proportional to the input holding current $I$ of the coil. ${ }^{(11)}$ Theoretically, from the force balance equation, it is apparent that $I=80 \mathrm{~mA}$ is the maximum holding current $I$ when the VCM actuator is at the upright position. It was also shown that the positioning repeatability for different positions is less than $40 \mu \mathrm{m} .^{(12)}$

\section{Mechanical Structure}

Figure 2 shows the structure of the proposed VCM actuator ${ }^{(10)}$ to achieve the following two major characteristics: small size and high positioning repeatability. The moving part comprises a lens module, a lens holder, and two permanent magnets. The fixed part comprises a Hall element, two coils, and two guide rods attached to a fixed base. One special feature of this design is that instead of the spring plates, two guide rods are used to maintain the trajectory of the lens holder to improve the drop impact resistance. The Hall element, which is principally used by motor drivers and for linear position detection, can detect the magnetic field and output a voltage signal proportional to the intensity of the magnetic flux density.

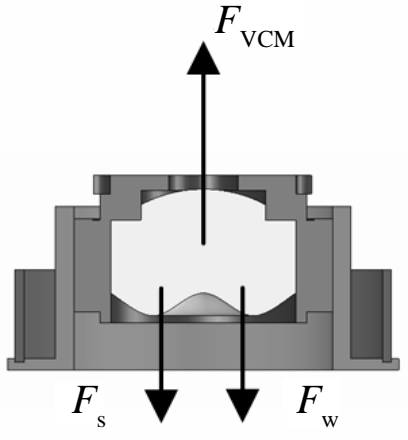

(a)

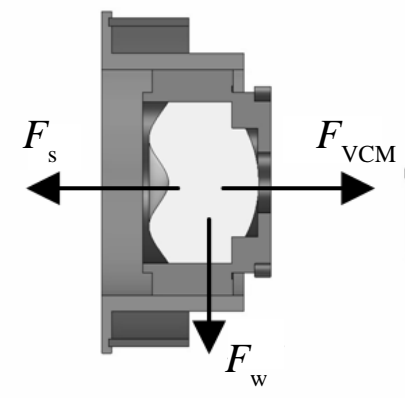

(b)

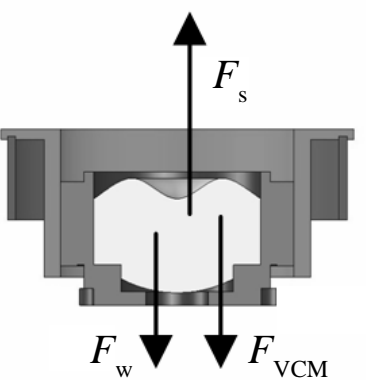

(c)

Fig. 1. Simplified force models of a conventional VCM ((a) upright, (b) horizontal, (c) upsidedown). 


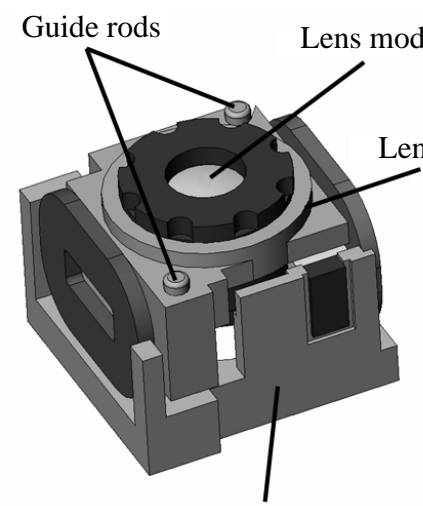

Fixed base

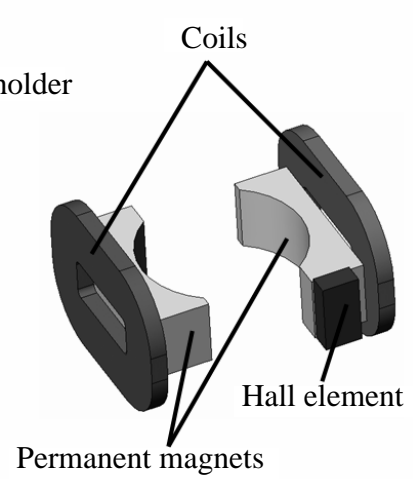

(b)

Fig. 2. (a) Structure of the proposed $\operatorname{VCM}^{(10)}$ and (b) the relative position of coils, permanent magnets and the Hall element.

\section{Electromagnetic Design}

The finite element method is one of the typical methods used for the electromagnetic analysis of the motor design. To reduce the size and improve the positioning repeatability, the design parameters (e.g., the dimensions of the permanent magnets and coils) are varied to determine the best design. Table 1 shows a list of the selected values of the design parameters of the proposed VCM actuator.

The Hall element, which is used to provide the position feedback signals, is mounted on one side of a permanent magnet parallel to the $x z$ plane to sense the magnetic flux density along the $y$-axis of the permanent magnet. To successfully obtain a closed-loop position control, it is important to linearly vary the magnetic flux density with $\delta$. Figure 3 shows the calculated magnetic flux density of the VCM actuator. We can extract the magnetic flux densities along the side, where the Hall element is placed, to verify the linearity of the VCM actuator. From this figure, we observe that the magnetic flux density is a linear function of $\delta$. According to the specifications of the Hall element and Fig. 4, we can theoretically obtain the resolution (voltage/displacement) of the output voltage of the Hall element as $2.35 \mathrm{mV} / 0.01 \mathrm{~mm}$. Furthermore, we also built a prototype (Fig. 6) of the proposed VCM actuator and measured the resolution, which is $2.29 \mathrm{mV} / 0.01 \mathrm{~mm}$, of the output voltage of the Hall element. Both the calculated and measured resolutions match very well. These data confirm that this design can achieve a high positioning accuracy for autofocusing. 
Table 1

Design parameters of the proposed VCM actuator.

\begin{tabular}{lc}
\hline Variable & Corresponding value \\
\hline Height of permanent magnet & $1.6 \mathrm{~mm}$ \\
Length of permanent magnet & $4.1 \mathrm{~mm}$ \\
Thickness of permanent magnet & $0.5 \mathrm{~mm}$ \\
Height of coil & $3.7 \mathrm{~mm}$ \\
Length of coil & $5.3 \mathrm{~mm}$ \\
Thickness of coil & $0.4 \mathrm{~mm}$ \\
Gap width between permanent magnet & $0.2 \mathrm{~mm}$ \\
\hline
\end{tabular}

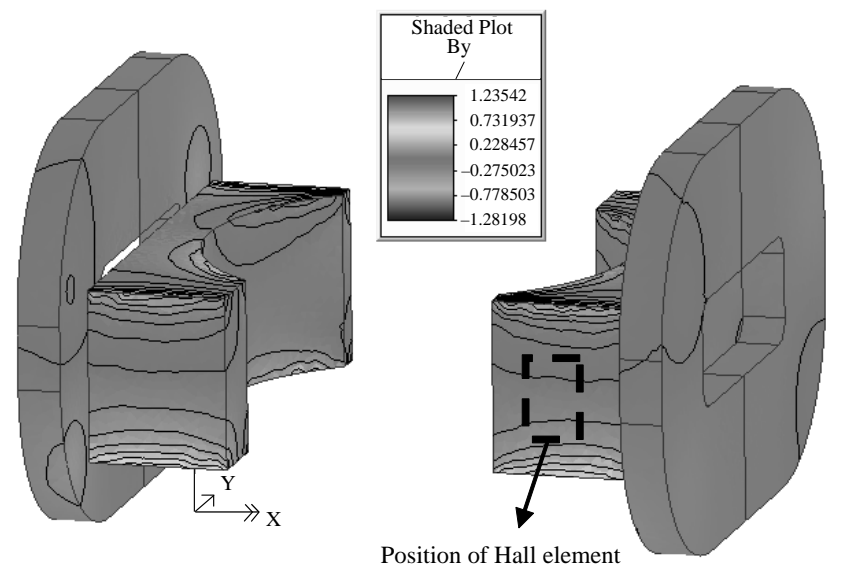

Fig. 3. Magnetic flux density of the proposed VCM actuator in $y$-axis direction.

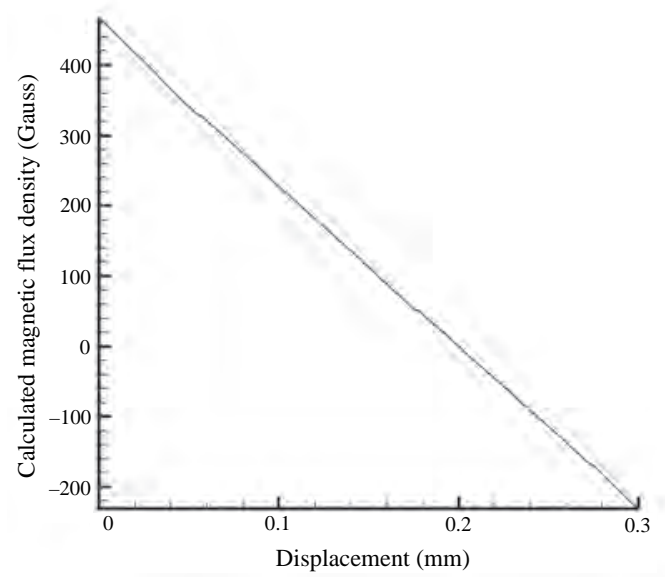

Fig. 4. Sensed magnetic flux density versus the displacement $\delta$. 


\section{Electronic Circuit Design}

The overall control block diagram of the proposed actuator is depicted in Fig. 5. This feedback control system can be separated into five parts: a voltage command output unit, a proportional plus integral plus derivative (PID) controller, a VCM driver, a VCM actuator, and a Hall signal processing unit. The voltage command output unit consists of a single chip (8051) and a D/A converter (DAC5571). The chip generates a digital $\mathrm{I}^{2} \mathrm{C}$ signal proportional to the displacement command. The D/A converter then converts the generated $\mathrm{I}^{2} \mathrm{C}$ signal to a command voltage $V_{\text {command }}\left(0 \mathrm{~V}<V_{\text {command }}<3.3 \mathrm{~V}\right)$. The Hall signal processing unit consists of a Hall element and a signal processing circuit. The output voltage $V_{\text {out }}$ of the Hall element is proportional to the magnetic flux density and working voltage. To limit $V_{\text {out }}$ within the linear region of the Hall element, the working voltage of the Hall element must be controlled to ensure that $V_{\text {out }}$ is less than $150 \mathrm{mV}$ at any displacement. Then, $V_{\text {out }}$ is filtered and amplified through the signal processing circuit to obtain $V_{\text {Hall }}$ in the range of $0 \mathrm{~V} \leq V_{\text {Hall }} \leq 3.3 \mathrm{~V}$. Therefore, in this feedback path, the Hall element can output a voltage $V_{\text {Hall }}$ proportional to $\delta$. The control voltage is obtained from the integration of the difference $V_{\text {error }}$ between $V_{\text {Hall }}$ and the $V_{\text {command }}$ supplied by the voltage command output unit. If the error signal $V_{\text {error }}$ converges to zero, it means that the moving part of the proposed actuator reaches the targeted displacement. If $V_{\text {error }}$ is not zero, the PID controller generates the control law for the VCM driver to tune the displacement of the moving part until the targeted displacement is achieved.

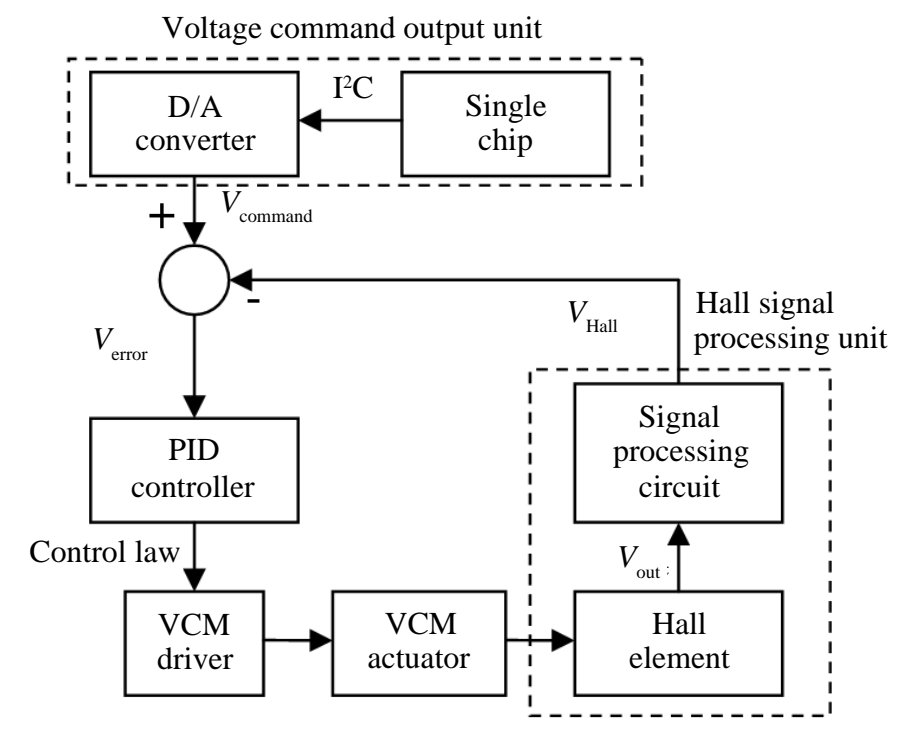

Fig. 5. Control block diagram of the proposed actuator. 


\section{Positioning Repeatability}

The VCM actuator was validated by constructing a prototype (Figs. 6 and 7). To test its positioning repeatability, a noncontacting laser displacement meter (Keyence, LC-2440) is used to measure the displacement of the moving part. Figures 8 and 9 show the output displacement versus the input displacement command of the moving part under three different postures (i.e., vertical, horizontal, and $45^{\circ}$ ) by repeating the displacement command back and forth for five times in each figure. It can be estimated that owing to the characteristics of the closed-loop position control, the positioning repeatability of the prototype is less than $5 \mu \mathrm{m}$. As compared with the conventional VCM actuator with the open-loop position control, the proposed VCM actuator can improve the positioning repeatability by $87.5 \%$ of the typical value.

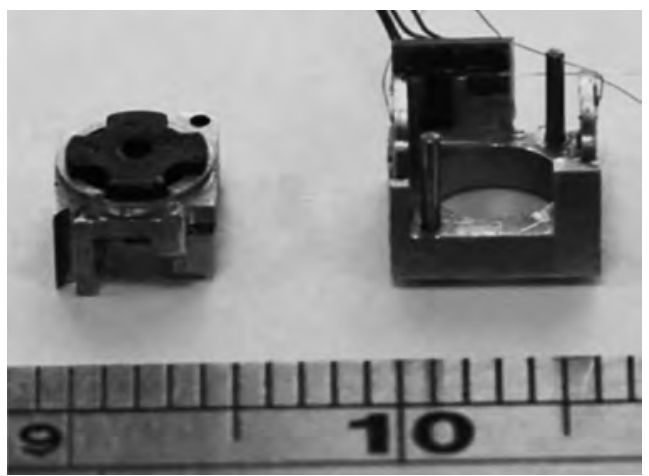

Fig. 6. Photograph of laboratory-built VCM actuator.

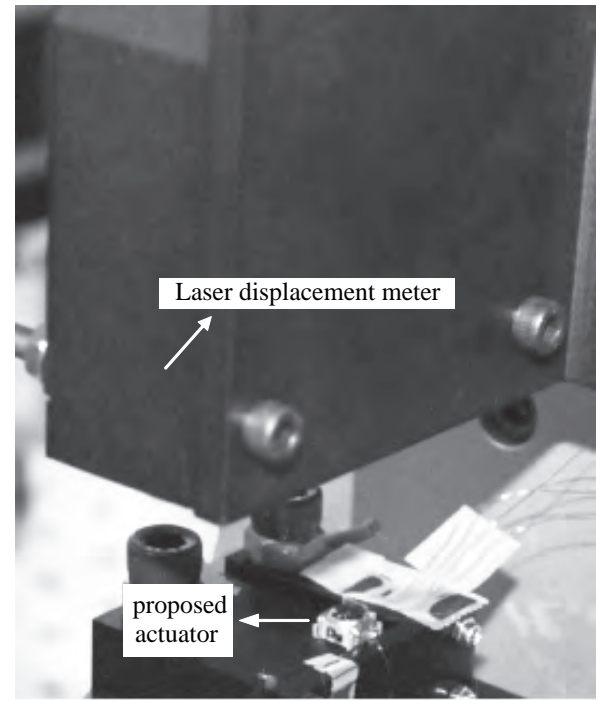

Fig. 7. Experimental setup. 


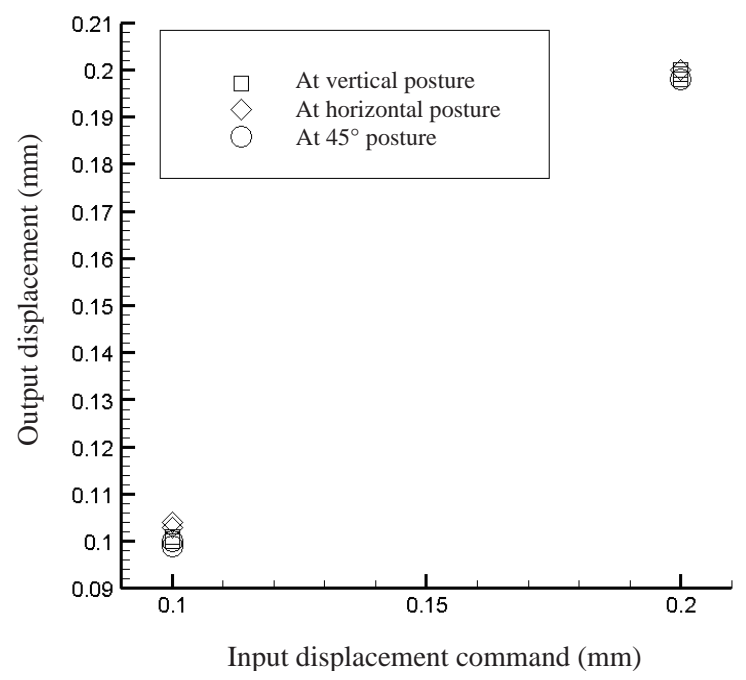

Fig. 8. Output displacement versus the input displacement command (from $\delta=0.1 \mathrm{~mm}$ to $\delta=0.2$ $\mathrm{mm}$ ) of the moving part under three different postures.

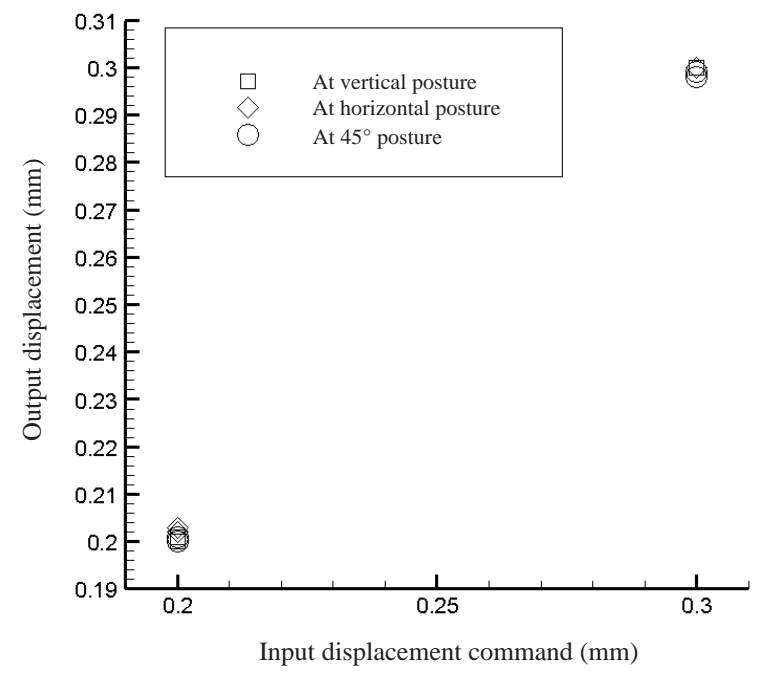

Fig. 9. Output displacement versus the input displacement command (from $\delta=0.2 \mathrm{~mm}$ to $\delta=0.3$ $\mathrm{mm}$ ) of the moving part under three different postures. 


\section{Conclusion}

We successfully developed a miniature autofocusing actuator (size: $\left.6.5 \times 6.5 \times 4 \mathrm{~mm}^{3}\right)^{(10)}$ using a VCM to improve the positioning repeatability of a portable autofocusing camera module. By using a novel configuration and closed-loop position control, the positioning repeatability of the proposed actuator is improved to $5 \mu \mathrm{m}$ independent of its position. These facts prove that as compared with the existing VCM actuators, the proposed VCM actuator is small and has a high positioning repeatability.

\section{Acknowledgment}

This study was supported by the Ministry of Economic Affairs of Taiwan. The authors would like to express special thanks to the Laser Application Technology Center, Industrial Technology Research Institute, for technologic assistance.

\section{References}

1 S. M. Sohn, S. H. Yang, S. W. Kim, K. H. Baek and W. H. Paik: IEEE Trans. Consum. Electron. 52 (2006) 10.

2 C. W. Chiu, P. C.-P. Chao and D. Y. Wu: IEEE Trans. Magn. 43 (2007) 2582.

3 M. J. Chung: Proc. SPIE 6048 (2005) p. 60480J.

4 H. P. Ko, S. Kim, S. N. Borodinas, P. E. Vasiljev, C. Y. Kang and S. J. Yoon: Sens. Actuators A 125 (2006) 477.

5 Y. J. Chang, K. Mohseni and V. M. Bright: Sens. Actuators A 136 (2007) 546.

6 S. Manabu and Y. Morimasa: Japan Patent 2002-365514 (2002).

7 S. M. Jang, J. Y. Choi, S. H. Lee, H. W. Cho and W. B. Jang: IEEE Trans. Magn. 40 (2004) 2068.

8 Y. Hirano and J. Naurse: IEEE Trans. Magn. 25 (1989) 3073.

9 H. C. Yu, T. Y. Lee, S. K. Lin, L. T. Kuo, S. J. Wang, J. J. Ju and D. R. Huang: J. Appl. Phys. 99 (2006) 08R901.

10 C. S. Liu and P. D. Lin: Opt. Express 16 (2008) 2533.

11 J. C. Ryu and Y. H. Cho: Sens. Mater. 19 (2007) 107.

12 http://www.shicoh.com/e/index.ht. 